\title{
Identificação e caracterização físico-química de frutos de laranja de Russas no semiárido cearense, Brasil
}

\author{
Kássio Ewerton Santos Sombra ${ }^{1}$, Alexandre Caique Costa e Silva ${ }^{2}$, \\ Ana Janaina Oliveira Rodrigues ${ }^{3}$, Francisco Leandro Costa Loureiro ${ }^{4}$, \\ Cleilson do Nascimento Uchôa ${ }^{2} \&$ Pahlevi Augusto de Souza ${ }^{5}$
}

\section{RESUMO}

A laranja de Russas é uma variedade de laranja doce (Citrus sinensis L. Osbeck) (Rutaceae), historicamente propagada por semente e selecionada empiricamente por agricultores familiares, apresenta inúmeras mutações com características diferenciadas, de maturação precoce e alta aceitabilidade. $\mathrm{O}$ trabalho teve objetivo de identificar e caracterizar a qualidade físico-química de frutos de laranjeiras de Russas pés francos, comparando-os com frutos do clone de laranja BRS Russas 02, enxertada sobre citrandarin San Diego (Citrus sunki Hayata hort. ex Tanaka x Poncirus trifoliata L. Raf.) (Rutaceae). Foram identificados frutos de sete laranjeiras pés francos, nomeando-os de acordo com a propriedade de coleta como laranjeira Rosa, Silva Neto, Doce do córrego, Catita, Tatá, Alves e Melancias. Ao realizar a comparação, constatou-se que os frutos da laranjeira Alves apresentavam maiores diâmetros longitudinais e transversais, maior massa de fruto, maior massa de suco e rendimento, predominando o formato redondo uniforme. Já os frutos da laranjeira Rosa continham maior número de sementes, enquanto os da laranjeira Silva Neto continham maior teor de sólidos solúveis ( ${ }^{\circ}$ Brix), menor acidez e maior conteúdo de vitamina $\mathrm{C}$, além dos índices de maturidade e tecnológico mais elevados entre as laranjeiras pés francos, se enquadrando como alternativa para clonagem e melhoramento, e satisfazer consumidores de citros de mesa, o que pode impulsionar a citricultura no semiárido.

Termos de indexação: agricultura familiar, citricultura, laranja doce, pós-colheita.

\section{Identification and physical-chemical characterization of Russas orange fruits in the semi-arid of Ceará, Brazil}

\section{SUMMARY}

The Russas orange is a variety of sweet orange (Citrus sinensis L. Osbeck) (Rutaceae), historically propagated by seed and selected empirically by family farmers, presents numerous mutations with differentiated characteristics, of early maturation and high acceptability. The objective of this work was to identify and characterize the physico-chemical quality of fruits of Russas

\footnotetext{
${ }^{1}$ Departamento de Fitotecnia, Universidade Federal do Ceará - UFC, Fortaleza, CE, Brasil

${ }^{2}$ Departamento de Agronomia, Instituto Federal de Educação, Ciência e Tecnologia do Ceará - IFCE, Fortaleza, CE, Brasil

${ }^{3}$ Departamento de Engenharia Agrícola, Universidade Federal do Ceará - UFC, Fortaleza, CE, Brasil

${ }^{4}$ Departamento de Fitotecnia, Universidade Federal Rural do Semiárido - UFERSA, Mossoró, RN, Brasil

${ }_{5}^{5}$ Departamento de Agronomia, Instituto Federal de Educação, Ciência e Tecnologia do Rio Grande do Norte - IFRN, Currais Novos, RN, Brasil
}

Autor correspondente: Kássio Ewerton Santos Sombra, Departamento de Fitotecnia, Universidade Federal do Ceará - UFC, Av. Mister Hull, 2977, Bloco 805, Campus do Pici, CEP 60356001, Fortaleza, CE, Brasil. E-mail: kassiosombra@gmail.com 
orange, propagated by seeds, comparing them with fruits of BRS Russas 02 orange clone grafted on citrandarin San Diego (Citrus sunki Hayata hort. ex Tanaka x Poncirus trifoliate L. Raf.) (Rutaceae). Were identified fruits of seven oranges seed-propagated, naming them according to the property of collection as orange tree Rosa, Silva Neto, Sweet of the stream, Catita, Tatá, Alves and Watermelons. In the comparison, it was found that the fruits of the orange Alves presented larger longitudinal and transverse diameters, greater fruit mass, greater mass of juice and yield, predominating the uniform round shape. On the other hand, the fruits of the orange tree Rosa contained more seeds, while those of the Silva Neto orange contained higher level of soluble solids ( ${ }^{\circ}$ Brix), lower acidity and higher vitamin $\mathrm{C}$ content, besides the higher maturity index and technological index among the orange fruits seed-propagated, being classified as an alternative for cloning and breeding, and satisfy consumers of table citrus, which may boost citrus cultivation in the semiarid.

Index terms: family farming, citriculture, sweet orange, post-harvest.

\section{INTRODUÇÃO}

A laranja de Russas é uma variedade de laranja doce (Citrus sinensis L. Osbeck) (Rutaceae), adaptada ao semiárido, que segundo Passos et al. (2013), possivelmente originou-se a partir da introdução de sementes de laranja doce por jesuítas portugueses, logo após o descobrimento no século XVI, sendo cultivada desta forma e selecionada ao longo de quatro séculos de forma empírica por figuras religiosas e agricultores familiares. Atingiu seu auge com a expansão da citricultura no começo do século $\mathrm{XX}$, quando a laranja de Russas era comercializada nos mercados locais do semiárido cearense, principalmente, para consumo in natura pela sua 'doçura' considerável, o que rendeu ao município de mesmo nome, o título de 'terra da laranja doce' (Passos et al., 2013; Sombra et al., 2016, 2018).

Apesar da importância socioeconômica da laranja de Russas para o semiárido cearense, pouco foi realmente investido, e diante da escassez de assistência técnica e extensão rural, a citricultura acabou apoiando-se no uso de mudas 'pés francos' e na irrigação por inundação total dos pomares. Práticas que contribuíram diretamente para o declínio da cadeia produtiva na região, entre os anos de 1974 e 1985, quando a 'gomose' causada por Phytophthora spp., proliferou-se e atingiu 90\% dos pomares constituídos da laranja doce altamente susceptível (Passos et al., 2013; Sombra et al., 2016, 2018).

As condições adversas induziram a seleção de plantas com alguma tolerância ou resistência, e apesar do declínio, o cultivo da laranja de Russas persistiu em pequenas e médias propriedades da agricultura familiar (Sombra et al., 2016, 2018).

Ao utilizar as sementes das laranjeiras remanescentes com caracteres desejáveis, como por exemplo, maior produtividade, frutos com maior tamanho, casca fina, menor número de sementes e/ou maior 'doçura' (relação doce/azedo), os agricultores selecionaram possíveis mutações, com potencial para atender consumidores de citros de mesa (Passos et al., 2013; Sombra et al., 2018). No entanto, carece de estudos que identifiquem e forneçam informações que amparem a seleção e a clonagem de plantas com características diferenciadas, capazes de atender as exigências do mercado consumidor (Passos et al., 2013; Rodrigues et al., 2015; Sombra et al., 2018).

Diante disto, o trabalho teve objetivo de identificar e caracterizar a qualidade físico-química de frutos de laranjeiras de Russas pés francos, comparando-os com frutos do clone de laranja BRS Russas 02.

\section{MATERIAL E MÉTODOS}

O trabalho foi realizado entre setembro de 2016 e fevereiro de 2017, e dividiu-se entre a identificação e coleta dos frutos cítricos em pomares produtivos inseridos na agricultura familiar dos municípios de Limoeiro do Norte, Quixeré e Russas, localizados na microrregião do Baixo Jaguaribe, e as determinações analíticas no Laboratório de Tecnologia de Pós-colheita do Instituto Federal de Educação, Ciência e Tecnologia do Ceará IFCE, em Limoeiro do Norte, CE, Brasil.

O clima do Baixo Jaguaribe classifica-se como semiárido (seco e muito quente), do tipo BSw'h' segundo classificação de 'Köppen' (Alvares et al., 2013). A temperatura média anual aproxima-se de $27,1^{\circ} \mathrm{C}$, oscilando entre $22,3^{\circ} \mathrm{C}$ e $34,8^{\circ} \mathrm{C}$, com umidade relativa (UR) do ar variando entre $44 \%$ e $85 \%$. A média do regime pluviométrico da microrregião é de $857,7 \mathrm{~mm}$, com distribuição irregular ao longo do ano, dividido em duas estações climáticas bem definidas, uma seca, que geralmente vai de junho 
a janeiro, e uma chuvosa, de fevereiro a maio (verão e outono) (INMET, 2017).

No Baixo Jaguaribe predominam 'Neossolos Flúvicos (RY)', considerados solos minerais não hidromórficos com alto potencial agrícola, principalmente quando apresentam textura 'média' e boa drenagem, porém, também se constatam solos de textura 'argilosa', principalmente em áreas de várzea, onde a vegetação nativa é formada basicamente por carnaubeiras (Copernicia prunifera Mill. H. E. Moore) (Arecaceae) intercaladas por pequenas propriedades, conduzidas em sua maioria com base na agricultura familiar (Guimaraes et al., 2018).

O delineamento experimental utilizado foi inteiramente casualizado (DIC), adotando quatro repetições por tratamento (laranjeira pé franco ou clone) e parcela útil de 8 frutos por repetição, no total de 32 frutos por tratamento. Os tratamentos consistiram de frutos de laranjeiras de Russas pés francos, comparando-os com frutos do clone de laranja BRS Russas 02 enxertada sobre citrandarin San Diego (Citrus sunki Hayata hort. ex Tanaka $\mathrm{x}$ Poncirus trifoliata L. Raf.) (Rutaceae).

A identificação de plantas pés francos da laranja de Russas (C. sinensis) que produziam frutos com características agronômicas desejáveis baseou-se nas informações fornecidas pelos agricultores, obtidas em visitas técnicas realizadas a alguns dos principais pomares cítricos produtivos. A coleta de dados foi realizada por observação direta intensiva através de entrevista estruturada, auxiliada por um roteiro previamente elaborado, que listava características como, por exemplo, produção anual, tempo entre florescimento e a colheita, comercialização e aceitação dos frutos (Lakatos \& Marconi, 2017; Marconi \& Lakatos, 2017; Sombra et al., 2018). A seleção buscou plantas com idade entre 10 e 30 anos, em pomares com espaçamento médio de $7 \times 7 \mathrm{~m}$, que geralmente, realizavam adubações orgânicas de cobertura com estercos e restos vegetais, coroamento e cobertura morta, indução de florescimento por estresse hídrico, eliminação de brotos 'ladrões' e irrigação por superfície (Sombra et al., 2018).

Já os frutos do clone de laranja BRS Russas 02 (C. sinensis) foram obtidos de uma planta enxertada sobre citrandarin San Diego (C. sunki x P. trifoliata) com 3 anos de idade, localizada no pomar experimental JRSN, implantado na agricultura familiar pela Empresa Brasileira de Pesquisa Agropecuária (Embrapa) na localidade de Córrego da Catita, zona rural do município de Russas, Ceará. O pomar é constituído por 130 plantas cítricas (combinações copa/porta-enxerto), espaçadas $5 \times 2 \mathrm{~m}$, adubadas com esterco bovino e irrigadas por inundação das microbacias.

A colheita dos frutos foi realizada manualmente de forma aleatória, a meia altura (em média $2 \mathrm{~m}$ ) nos quatro quadrantes da copa, acondicionando-os em sacos de papel e transportando-os em bolsa térmica, logo em seguida, ao laboratório, onde foram realizadas as determinações analíticas.

As variáveis físicas consistiram de aparência externa, massa do fruto, diâmetro longitudinal e transversal, formato do fruto, números de sementes e espessura da casca.

A aparência externa (AE) foi determinada através de escala de notas que variaram de 5 a 0 , de acordo com o aspecto geral e severidade das injúrias, observando e mensurando a intensidade de possíveis depressões, manchas e/ou danos a integridade dos frutos, considerando-se impróprios para comercialização frutos com nota inferior a 3 (Rocha et al., 2005). A massa do fruto (MF), em g, foi determinada por pesagem individual em balança digital analítica $(0,001 \mathrm{~g})$, enquanto os diâmetros longitudinais (DL), em mm, e transversais (DT), em $\mathrm{mm}$, foram mensurados com auxílio de paquímetro digital $(0,01 \mathrm{~mm})$, utilizando os valores para cálculo e determinação do formato do fruto, DT/DL.

Logo após, partiram-se os frutos transversalmente para contagem do número de sementes (SF) e mensuração da espessura da casca (EC), em mm, com paquímetro digital (Agostini, 2012). Em seguida, extraíram-se os sucos com espremedor elétrico e determinou-se a massa do suco (MS), em g, calculando-se o rendimento (RS), em \%, através da fórmula (AOAC, 2016):

$R S=(M S / M F) \times 100$

A caracterização química foi realizada com base nas amostras de suco, adotando-se triplicata em todas as análises. As variáveis químicas consistiram no potencial hidrogeniônico $(\mathrm{pH})$, sólidos solúveis, acidez titulável, Ratio, vitamina $\mathrm{C}$ total e índice tecnológico.

$\mathrm{O} \mathrm{pH}$ foi determinado utilizando potenciômetro digital de bancada. Os sólidos solúveis (SS) foram mensurados por leitura direta em refratômetro digital, com escala de 0 a $95^{\circ}$ Brix e compensação automática de temperatura, obtendo-se valores com precisão de $0,1{ }^{\circ}$ Brix à $25^{\circ} \mathrm{C}$, conforme metodologia descrita por IAL (2008).

A acidez titulável (AT) foi determinada em ácido orgânico, utilizando $1 \mathrm{~g}$ de suco diluído em $50 \mathrm{~mL}$ de água destilada e fenolftaleína (1\%) como indicador, titulando-se com hidróxido de sódio $(\mathrm{NaOH})$ 0,1 M, e obtendo-se resultados em $\mathrm{g}$ de ácido cítrico/ $100 \mathrm{~g}$ de suco 
(AOAC, 2016). O Ratio (SS/AT) foi calculado com base na relação entre os valores de 'SS' e 'AT' (IAL, 2008).

A vitamina $\mathrm{C}$ total foi determinada pelo método colorimétrico, utilizando $5 \mathrm{~g}$ de suco e solução de Tillman (DFI - 2,6 diclofenolindofenol 0,02\%) para titulação, obtendo resultados em mg de ácido ascórbico em $100 \mathrm{~g}$ de suco (AOAC, 2016). Por último, calculou-se o índice tecnológico (IT), definido como quantidade de sólidos solúveis por caixa de 40,8 $\mathrm{kg}$ de frutos ( $\mathrm{kg}$ de SS. caixa ${ }^{-1}$ ), conforme a formula de Di Giorgi (1993):

$I T=(R S \times S S \times 40,8) / 10.000$

Os dados foram submetidos à análise de variância (ANOVA), comparando-se as médias em casos de diferença significativa através do Teste de Scott-Knott ao nível de $5 \%$ de probabilidade $(\mathrm{p} \leq 0,05)$, além de calcular também o desvio padrão de cada amostra, utilizando-se auxílio do software estatístico SAS ${ }^{\circledR}$ (SAS Institute, 2017).

\section{RESULTADOS E DISCUSSÃO}

A aparência externa (AE) dos frutos da laranjeira Silva Neto e os do clone BRS Russas 02 obtiveram as maiores notas de AE, com 4,3 e 4,4; respectivamente. Entre os demais frutos, os das laranjeiras Doce do Córrego e Rosa, obtiveram notas superiores a 4 de AE, enquanto os frutos das laranjeiras Catita, Tatá e Melancias, obtiveram as menores notas, de 3,3; 3,6 e 3,3, respectivamente (Tabela 1 ).
Os frutos com nota superior a 4 apresentavam qualidade fenotípica, com tamanho, forma e cores uniformes, além da ausência de desordens mecânicas, fisiológicas e/ou patológicas que pudessem ser observadas na análise visual, consideradas por Abouzari \& Nezhad (2016), as principais características responsáveis pela qualidade física das frutas cítricas, além de serem base para a aceitabilidade pelo consumidor (Silva Júnior et al., 2010).

Os frutos da laranjeira Alves apresentavam maiores DL e DT, como observa-se nas médias de $78,49 \mathrm{~mm}$ e $76,94 \mathrm{~mm}$, respectivamente, classificados como 'grandes' (maior que $71 \mathrm{~mm}$ ) de acordo com a classificação dos citros de mesa (CEAGESP, 2011). Os menores frutos foram registrados nas laranjeiras Rosa e Silva Neto, com DL de 64,22 mm e 64,05 mm; e DT de 67,24 mm e $64,83 \mathrm{~mm}$, respectivamente (Tabela 1 ).

A partir dos DL e DT, calculou-se e determinou-se o formato dos frutos, obtendo-se valores entre 0,97 (clone de laranja BRS Russas 02) e 1,07 (laranjeira Tatá). Já os frutos das laranjeiras de Russas pés francos obtiveram valores entre 0,98 (Alves) e 1,07 (Tatá), predominando o formato arredondado, com alta similaridade entre os diâmetros longitudinais e transversais.

Os frutos da laranjeira Alves apresentavam maior MF, atingindo média de $243,17 \mathrm{~g}$, diferindo estatisticamente dos frutos do clone de laranja BRS Russas, que apresentavam média de $212,50 \mathrm{~g}$. Os frutos de menor massa foram os das laranjeiras Rosa (163,36 g), Silva Neto $(149,56 \mathrm{~g})$ e Catita $(169,69 \mathrm{~g})$ (Tabela 2).

Estes resultados são superiores aos obtidos por Cavalcante et al. (2006), para dezoito variedades de

Tabela 1. Aparência externa (AE), diâmetro longitudinal (DL), diâmetro transversal (DT) e formato do fruto (DT/DL), Limoeiro do Norte, Ceará - Brasil. 2018.

\begin{tabular}{lcccc}
\hline \multicolumn{1}{c}{ Tratamento } & AE & DL $(\mathrm{mm})$ & DT $(\mathrm{mm})$ & DT/DL \\
\hline Rosa & $4,1 \pm 0,36 \mathrm{~b}$ & $64,22 \pm 2,35 \mathrm{e}$ & $67,24 \pm 2,88 \mathrm{c}$ & $1,0472 \pm 0,043 \mathrm{~b}$ \\
Silva Neto & $4,3 \pm 0,45 \mathrm{a}$ & $64,05 \pm 2,10 \mathrm{e}$ & $64,83 \pm 1,97 \mathrm{~d}$ & $1,0131 \pm 0,019 \mathrm{c}$ \\
Doce do Córrego & $4,1 \pm 0,28 \mathrm{~b}$ & $71,05 \pm 4,22 \mathrm{c}$ & $71,34 \pm 4,59 \mathrm{~b}$ & $1,0040 \pm 0,029 \mathrm{c}$ \\
Catita & $3,3 \pm 0,45 \mathrm{c}$ & $66,84 \pm 1,93 \mathrm{~d}$ & $68,84 \pm 2,50 \mathrm{c}$ & $1,0301 \pm 0,026 \mathrm{~b}$ \\
Tatá & $3,6 \pm 0,82 \mathrm{c}$ & $67,34 \pm 4,54 \mathrm{~d}$ & $72,22 \pm 5,23 \mathrm{~b}$ & $1,0733 \pm 0,032 \mathrm{a}$ \\
Alves & $3,8 \pm 0,34 \mathrm{~b}$ & $78,49 \pm 3,09 \mathrm{a}$ & $76,94 \pm 2,88 \mathrm{a}$ & $0,9806 \pm 0,013 \mathrm{~d}$ \\
Melancias & $3,3 \pm 0,55 \mathrm{c}$ & $69,71 \pm 3,55 \mathrm{c}$ & $69,40 \pm 3,25 \mathrm{c}$ & $0,9961 \pm 0,024 \mathrm{c}$ \\
BRS Russas 02 & $4,4 \pm 0,60 \mathrm{a}$ & $74,91 \pm 3,55 \mathrm{~b}$ & $72,53 \pm 2,09 \mathrm{~b}$ & $0,9702 \pm 0,043 \mathrm{~d}$ \\
C.V. (\%) & 16,42 & 6,23 & 6,06 & 3,46 \\
Valor de F & $9,180^{* *}$ & $27,627 * *$ & $15,069 * *$ & $19,438^{* *}$ \\
Valor-p & 0,0000 & 0,0000 & 0,0000 & 0,0000 \\
\hline
\end{tabular}

As médias seguidas pela mesma letra não diferem estatisticamente entre si; ** significativo ao nível de $1 \%$ de probabilidade (Scott-Knott, $\mathrm{p}<0,01$ ). 
Tabela 2. Espessura da casca (EC), número de sementes por fruto (SF), massa do fruto (MF) e massa do suco (MS), Limoeiro do Norte, Ceará - Brasil. 2018.

\begin{tabular}{lcrcc}
\hline \multicolumn{1}{c}{ Tratamento } & EC (mm) & SF (unid.) & MF $(\mathrm{g})$ & MS $(\mathrm{g})$ \\
\hline Rosa & $2,83 \pm 0,3 \mathrm{c}$ & $16,3 \pm 2,0 \mathrm{a}$ & $163,36 \pm 17,69 \mathrm{~d}$ & $82,11 \pm 9,66 \mathrm{c}$ \\
Silva Neto & $2,96 \pm 0,3 \mathrm{c}$ & $13,4 \pm 2,8 \mathrm{~b}$ & $149,56 \pm 13,29 \mathrm{~d}$ & $73,81 \pm 8,07 \mathrm{~d}$ \\
Doce do Córrego & $3,95 \pm 0,5 \mathrm{a}$ & $9,7 \pm 3,1 \mathrm{c}$ & $190,35 \pm 33,48 \mathrm{c}$ & $87,68 \pm 15,66 \mathrm{c}$ \\
Catita & $2,81 \pm 0,3 \mathrm{c}$ & $13,8 \pm 3,5 \mathrm{~b}$ & $169,69 \pm 15,76 \mathrm{~d}$ & $83,74 \pm 9,12 \mathrm{c}$ \\
Tatá & $3,35 \pm 0,4 \mathrm{~b}$ & $10,6 \pm 2,8 \mathrm{c}$ & $181,47 \pm 23,40 \mathrm{c}$ & $71,45 \pm 9,17 \mathrm{~d}$ \\
Alves & $4,32 \pm 0,4 \mathrm{a}$ & $12,0 \pm 2,4 \mathrm{c}$ & $243,17 \pm 25,69 \mathrm{a}$ & $119,58 \pm 15,03 \mathrm{a}$ \\
Melancias & $4,04 \pm 0,4 \mathrm{a}$ & $11,8 \pm 2,4 \mathrm{c}$ & $181,55 \pm 23,40 \mathrm{c}$ & $77,92 \pm 15,49 \mathrm{~d}$ \\
BRS Russas 02 & $3,52 \pm 0,4 \mathrm{~b}$ & $3,0 \pm 1,9 \mathrm{~d}$ & $212,50 \pm 19,15 \mathrm{~b}$ & $108,40 \pm 8,65 \mathrm{~b}$ \\
C.V. $(\%)$ & 16,03 & 30,76 & 15,76 & 17,65 \\
Valor de F & $22,142^{* *}$ & $25,620^{* *}$ & $30,394 * *$ & $24,164^{* *}$ \\
Valor-p & 0,0000 & 0,0000 & 0,0000 & 0,0000 \\
\hline
\end{tabular}

As médias seguidas pela mesma letra não diferem estatisticamente entre si; $* *$ significativo ao nível de $1 \%$ de probabilidade (Scott-Knott, $\mathrm{p}<0,01$ ).

laranja, além de Lemos et al. (2012), para frutos de laranjeiras Pera, e Portella (2015), para laranjas Bahia, Seleta, Lima e Folha murcha, com predominância de frutos com tamanhos 'pequeno' ou 'médio', que em sua maioria acabam destinados à indústria de suco congelado.

As cascas com maior espessura foram registradas nos frutos das laranjeiras Doce do Córrego, Alves e Melancias, com médias de 3,95mm, 4,32mm e 4,04mm, enquanto os frutos das laranjeiras Rosa, Silva Neto e Catita, continham cascas finas, com 2,83 mm, 2,96 mm e $2,85 \mathrm{~mm}$, respectivamente (Tabela 2).

Os frutos de casca 'grossa' e tamanho grande, como os da laranjeira Alves, podem acabar sendo pouco atrativas ao consumo in natura, e devido à maior massa e rendimento de suco, apresentam enorme potencial para a industrialização, seja na forma de suco concentrado congelado, minimamente processado ou beneficiado na forma de doces ou compotas, por exemplo.

Os frutos da laranjeira Rosa continham maior número SF, com 16 sementes em média, seguidos dos frutos da laranjeira Catita, com 10 SF (Tabela 2). O número de SF nas laranjas Rosa e Catita foram superiores aos comumente obtidos em cultivares comerciais como Pera ou Hamlin, que apresentam em média 5 a 6 SF (CEAGESP, 2011). O maior número de SF pode dificultar a comercialização, principalmente para consumo in natura, porém, ao considerar a possibilidade de uso da laranja de Russas como porta-enxerto, o número de SF pode ser um caractere desejável, como evidenciaram Soares Filho et al. (2002) ao registrarem em média 26,3 SF de laranjeira azeda
(C. aurantium L.); 16,6 SF de tangerineira Clementina (C. clementina) e 5,7 SF de tangerineira Sunki (C. sunki Hort. ex Tan.).

Todas os tratamentos apresentaram RS superior a $35 \%$, destacando-se os frutos das laranjeiras Rosa, Silva Neto, Catita e Alves, com rendimento médio de 50,32\%, $49,30 \%, 49,31 \%$ e 49,21\%, respectivamente (Tabela 3), atendendo as exigências para o mercado de frutos in natura, que segundo Salibe (1977), devem aproximar-se de 35\%, assim como, indústria, que segundo Koller (1994), devem aproxima-se de $40 \%$. Rendimentos similares aos obtidos por Amorim et al. (2014), em frutos de laranjeira Pera Rio sobre porta-enxertos híbridos de trifoliata, com média de $51,5 \%$ de suco.

Os frutos da laranjeira Silva Neto destacaram-se com maior teor SS, atingindo média de $14,77^{\circ}$ Brix, diferindo estatisticamente das demais laranjeiras de Russas pés francos, que superaram facilmente os $11,8^{\circ}$ Brix apontado como média para a laranja Valência (Figueiredo et al., 2006), superando também os valores obtidos por Amorim et al. (2014) em frutos de laranja Pera Rio, de $8,86^{\circ}$ Brix de SS, porém, aproximando-se dos $13,6^{\circ}$ Brix obtidos por Cavalcante et al. (2006) para a variedade Hamlin.

As laranjeiras Silva Neto e Catita obtiveram os maiores índices tecnológicos, diferindo estatisticamente das demais laranjeiras, ou espécies cítricas, com IT de 2,97 kg de SS. caixa $^{-1}$ e 2,81 kg de SS. caixa ${ }^{-1}$, respectivamente. Valores superiores aos obtidos por Silva Júnior et al. (2010), que encontraram índices de $2,20 \mathrm{~kg}$ de SS. caixa ${ }^{-1}$ para laranja Valência 718 e 2,16 kg de SS. caixa ${ }^{-1}$ para Valência. 
Tabela 3. Rendimento do suco (RS), sólidos solúveis (SS) e índice tecnológico (IT) dos frutos, Limoeiro do Norte, Ceará - Brasil. 2018.

\begin{tabular}{lccc}
\hline \multicolumn{1}{c}{ Tratamento } & RS $(\%)$ & SS $\left({ }^{\circ}\right.$ Brix $)$ & IT $\left(\mathrm{kg} \mathrm{de} \mathrm{SS} \mathrm{caixa}^{-1}\right)$ \\
\hline Rosa & $50,32 \pm 2,35 \mathrm{a}$ & $12,86 \pm 0,31 \mathrm{c}$ & $2,6397 \pm 0,06 \mathrm{~b}$ \\
Silva Neto & $49,30 \pm 2,34 \mathrm{a}$ & $14,77 \pm 0,21 \mathrm{a}$ & $2,9713 \pm 0,06 \mathrm{a}$ \\
Doce do Córrego & $46,26 \pm 2,96 \mathrm{a}$ & $12,74 \pm 0,15 \mathrm{c}$ & $2,4954 \pm 0,05 \mathrm{c}$ \\
Catita & $49,31 \pm 1,85 \mathrm{a}$ & $14,00 \pm 0,21 \mathrm{~b}$ & $2,8155 \pm 0,04 \mathrm{a}$ \\
Tatá & $39,37 \pm 3,82 \mathrm{c}$ & $14,06 \pm 0,05 \mathrm{~b}$ & $2,2701 \pm 0,04 \mathrm{c}$ \\
Alves & $49,21 \pm 4,01 \mathrm{a}$ & $12,60 \pm 0,15 \mathrm{c}$ & $2,5319 \pm 0,06 \mathrm{~b}$ \\
Melancias & $43,40 \pm 9,42 \mathrm{~b}$ & $13,06 \pm 0,53 \mathrm{c}$ & $2,3007 \pm 0,06 \mathrm{c}$ \\
BRS Russas 02 & $51,18 \pm 2,96 \mathrm{a}$ & $12,02 \pm 0,36 \mathrm{~d}$ & $2,5117 \pm 0,09 \mathrm{~b}$ \\
C.V. $(\%)$ & 11,17 & 2,80 & 4,94 \\
Valor de F & $14,953^{* *}$ & $24,402 * *$ & $20,812 * *$ \\
Valor-p & 0,0000 & 0,0000 & 0,000 \\
\hline
\end{tabular}

As médias seguidas pela mesma letra não diferem estatisticamente entre si; $* *$ significativo ao nível de $1 \%$ de probabilidade (Scott-Knott, $\mathrm{p}<0,01$ ).

Tabela 4. Valores de potencial hidrogeniônico (pH), acidez titulável (AT), Ratio (SS/AT) e vitamina C total dos frutos, Limoeiro do Norte, Ceará - Brasil. 2018.

\begin{tabular}{lcccc}
\hline \multicolumn{1}{c}{ Tratamento } & $\mathrm{pH}$ & $\mathrm{AT}(\mathrm{g} / 100 \mathrm{~g})$ & Ratio & $\begin{array}{c}\text { Vitamina C } \\
(\mathrm{mg} / 100 \mathrm{mg})\end{array}$ \\
\hline Rosa & $4,27 \pm 0,01 \mathrm{~b}$ & $0,53 \pm 0,01 \mathrm{c}$ & $24,23 \pm 1,36 \mathrm{c}$ & $61,71 \pm 2,50 \mathrm{~d}$ \\
Silva Neto & $4,26 \pm 0,11 \mathrm{~b}$ & $0,60 \pm 0,02 \mathrm{~b}$ & $24,67 \pm 1,11 \mathrm{c}$ & $71,35 \pm 1,41 \mathrm{~b}$ \\
Doce do Córrego & $4,32 \pm 0,17 \mathrm{~b}$ & $0,69 \pm 0,04 \mathrm{a}$ & $18,46 \pm 1,39 \mathrm{~d}$ & $67,70 \pm 1,75 \mathrm{c}$ \\
Catita & $4,34 \pm 0,08 \mathrm{~b}$ & $0,52 \pm 0,03 \mathrm{c}$ & $27,06 \pm 1,72 \mathrm{~b}$ & $47,37 \pm 2,11 \mathrm{e}$ \\
Tatá & $4,20 \pm 0,08 \mathrm{~b}$ & $0,57 \pm 0,03 \mathrm{~b}$ & $24,59 \pm 1,43 \mathrm{c}$ & $61,62 \pm 0,98 \mathrm{~d}$ \\
Alves & $4,51 \pm 0,02 \mathrm{a}$ & $0,46 \pm 0,01 \mathrm{~d}$ & $27,27 \pm 1,13 \mathrm{~b}$ & $43,16 \pm 3,12 \mathrm{e}$ \\
Melancias & $4,56 \pm 0,08 \mathrm{a}$ & $0,44 \pm 0,02 \mathrm{~d}$ & $30,07 \pm 2,97 \mathrm{a}$ & $46,96 \pm 3,08 \mathrm{e}$ \\
BRS Russas 02 & $4,40 \pm 0,01 \mathrm{a}$ & $0,46 \pm 0,02 \mathrm{~d}$ & $25,98 \pm 2,09 \mathrm{c}$ & $77,95 \pm 3,47 \mathrm{a}$ \\
C.V. $(\%)$ & 2,64 & 6,87 & 2,80 & 5,61 \\
Valor de F & $4,650 * *$ & $21,583^{* *}$ & $24,402 * *$ & $57,395 * *$ \\
Valor-p & 0,0021 & 0,0000 & 0,0000 & 0,0000 \\
\hline
\end{tabular}

As médias seguidas pela mesma letra não diferem estatisticamente entre si; ** significativo ao nível de $1 \%$ de probabilidade (Scott-Knott, $\mathrm{p}<0,01$ ).

Os menores índices foram registrados pelas laranjeiras Doce do Córrego, Tatá e Melancias, com 1,49 kg de SS. caixa $^{-1}, 1,27 \mathrm{~kg}$ de SS. caixa ${ }^{-1}$ e 1,30 kg de SS. caixa ${ }^{-1}$, respectivamente (Tabela 3 ).

Os menores valores de ácido cítrico foram observados nos frutos das laranjeiras Alves ( $0,46 \mathrm{~g}$ de ácido cítrico $\left.100 \mathrm{~g} \mathrm{suco}^{-1}\right)$ e Melancias $\left(0,44 \mathrm{~g}\right.$ de ácido cítrico $\left.100 \mathrm{~g} \mathrm{suco}{ }^{-1}\right)$, não diferindo estatisticamente do clone de laranja BRS Russas $02\left(0,46 \mathrm{~g}\right.$ de ácido cítrico $\left.100 \mathrm{~g} \mathrm{suco}{ }^{-1}\right)$, enquanto a maior acidez titulável foi observada nos frutos da laranjeira Doce do Córrego $\left(0,69 \mathrm{~g}\right.$ de ácido cítrico $\left.100 \mathrm{~g} \mathrm{suco}^{-1}\right)$ (Tabela 4). Ao comparar os resultados de acidez aos $0,75 \mathrm{~g}$ de ácido $100 \mathrm{~g} \mathrm{suco}^{-1}$ obtidos em frutos de laranjeiras Valência por Grizotto et al. (2012), pode-se inferir que, a menor acidez e os sólidos solúveis mais elevados da laranja de Russas, que superam cultivares cítricas comerciais, como Valência, Pera ou Hamlin (Figueiredo et al., 2006), se constitui como principal característica da variedade, inferindo sabor e aroma diferenciado aos frutos das laranjeiras de Russas.

Os maiores índices de maturação (Ratio), compreendidos como a razão entre o teor de sólidos solúveis (SS) e a acidez titulável (AT), foram registrados nos frutos da laranjeira Melancias, com Ratio de 30,07, diferindo estatisticamente 
dos frutos das demais laranjeiras, como, por exemplo, os frutos da laranjeira Alves $(27,27)$ ou Catita $(27,06)$. Valores próximos aos obtidos por Cavalcante et al. (2006) para as variedades de laranja João Nunes, Hamlin e Westin, de 23,46; 18,98 e 19,32 de índice de maturidade, respectivamente. Porém, os valores são superiores ao Ratio de 13,4 obtido por Auler et al. (2008) para a laranja Valência $718 \mathrm{em}$ avaliações realizadas no noroeste do Paraná.

Ao considerar que o Ratio representa a relação entre os açucares contidos nos sólidos solúveis e os ácidos inorgânicos (balanço do sabor doce/azedo), percebe-se a enorme importância desta característica no desenvolvimento, e consequentemente, na comercialização de laranjas doces (Silva Júnior et al., 2010).

Sendo assim, é possível inferir que os Ratios dos frutos de laranjeiras de Russas pés francos, por influenciarem diretamente a atratividade do produto, contribuem diretamente nas características dos frutos das variedades tradicionais de laranja doce, que destaca-se, geralmente, por sua 'doçura' elevada para o consumo in natura (Auler et al., 2008; Silva Júnior et al., 2010; Sombra et al., 2018).

Os frutos da laranjeira Silva Neto apresentavam maior conteúdo de vitamina $\mathrm{C}$, com 71,35 mg.100g de suco ${ }^{-1}$, resultado inferior ao dos frutos do clone de laranja BRS Russas 02, que apresentavam 77,95 mg.100g de suco $^{-1}$. Porém, todos os frutos apresentavam conteúdos de vitamina C total superiores ao nível mínimo proposto por Kimball (1991), de $50 \mathrm{mg} .100 \mathrm{~g}$ de suco ${ }^{-1}$.

Silva Júnior et al. (2010) obtiveram vitamina $\mathrm{C}$ total superior em frutos de laranja-da-terra, com média de 122,26 mg.100mg de suco-1 ${ }^{-1}$ assim como, $\mathrm{pH}$ médio de 5,33, superior ao $\mathrm{pH}$ médio dos frutos das mutações de laranja, que variou entre 4,20 dos frutos da laranjeira Tatá, e 4,51 dos frutos da laranjeira Alves.

Diante dos resultados elevados obtidos por algumas laranjeiras de Russas pés francos, como por exemplo, o índice tecnológico da laranjeira Silva Neto, que considera o rendimento de colheita $\left(\mathrm{kg} \mathrm{ha}^{-1}\right)$, o teor de sólidos solúveis ( ${ }^{\circ}$ Brix) e o rendimento do suco (\%); ou o conteúdo de vitamina $\mathrm{C}$ total, superior aos obtidos em variedades comerciais.

Assim, pode-se concluir que os frutos da laranja de Russas, podem, por exemplo, ser consumidos in natura ou na forma de suco concentrado, atendendo as diferentes exigências do mercado, tanto indústria de processamento, como consumidores de citros de mesa, desde que as plantas diferenciadas sejam devidamente identificadas, clonadas e disseminadas nos polos citrícolas nacionais, o que diante da rusticidade de cultivo da laranja de
Russas, pode impulsionar e diversificar a citricultura no semiárido brasileiro (Silva Júnior et al. 2010; Cunha Sobrinho et al., 2013).

\section{CONCLUSÕES}

O trabalho identificou sete laranjeiras de Russas pés francos, caracterizou e comparou a qualidade físico-química com a dos frutos do clone de laranja BRS Russas 02 . Dentre as laranjeiras de Russas pés francos, os frutos da laranjeira Alves apresentam maior diâmetro longitudinal e transversal, massa de fruto, massa e rendimento de suco, predominando o formato redondo uniforme.

Já os frutos da laranjeira Rosa apresentam maior número de sementes, enquanto os da laranjeira Silva Neto contém maior teor de sólidos solúveis ( ${ }^{\circ} \mathrm{Brix}$ ), menor acidez e maior conteúdo de vitamina $\mathrm{C}$, além dos índices de maturidade e tecnológicos mais elevados entre as laranjeiras pés francos da variedade tradicional de Russas, sendo alternativa para clonagem e melhoramento, como potencial de diversificar o mercado de citros de mesa, e contribuir com o desenvolvimento da citricultura no semiárido.

\section{AGRADECIMENTOS}

Ao Conselho Nacional de Desenvolvimento Científico e Tecnológico (CNPq) pela concessão de bolsas, à Embrapa Mandioca e Fruticultura Tropical, à Embrapa Semiárido e à Secretaria de Agricultura de Russas (SEAGRI).

\section{REFERÊNCIAS}

Abouzari, A., \& Nezhad, N. M. (2016). The investigation of citrus fruit quality Popular characteristic and breeding. Acta Universitatis Agriculturae et Silviculturae Mendelianae Brunensis, 64(3), 725-740.

Agostini, J. S. (2012) Caracterização, conservação póscolheita, processamento mínimo e adubação na qualidade de laranja 'Champagne' (Citrus sinensis L. Osbeck) (Tese de doutorado). Universidade Federal da Grande Dourados, Dourados.

Alvares, C. A., Stape, J. L., Sentelhas, P. C., Moraes, G., Leonardo, J., \& Sparovek, G. (2013). Köppen's climate 
classification map for Brazil. Meteorologische Zeitschrift, 22(6), 711-728.

Amorim, M. D. S., França, N. D. O., Soares Filho, W. S., Passos, O. S., \& Girardi, E. (2014) Desempenho inicial da Laranjeira pera sobre porta-enxertos híbridos de Trifoliata. In Proceedings of the 23th Congresso Brasileiro de Fruticultura (p. 1-4). Cuiabá.

Association of Official Analytical Chemists - AOAC. (2016). Official Methods of Analysis of AOAC International (20. ed., 3172 pp). Washington: AOAC.

Auler, P. A. M., Fiori-Tutida, A. C. G., \& Tazima, Z. H. (2008). Comportamento da laranjeira 'Valência' sobre seis porta-enxertos no noroeste do Paraná. Revista Brasileira de Fruticultura, 30(1), 229-234.

Cavalcante, Í. H. L., Martins, A. B. G., \& Stuchi, E. S. (2006). Fruit characteristics of eighteen orange cultivars. Revista de Biologia e Ciências da Terra, 6(2), 72-77.

Companhia de Entrepostos e Armazéns Gerais de São Paulo - CEAGESP. (2011). Normas de Classificação de Citros de Mesa/CEAGESP (11 pp.). São Paulo: CEAGESP.

Cunha Sobrinho, A. P., Magalhães, A. F. J., Souza, A. S., Passos, O. S., \& Soares Filho, W. S. (2013). Cultura do citros (399 pp.). Brasília: Embrapa Informação Tecnológica.

Di Giorgi, F. (1993). Qualidade da laranja para industrialização. Laranja, 11(1), 97-118.

Figueiredo, J. O. D., Negri, J. D. D., Mattos Junior, D. D., Pio, R. M., Azevedo, F. A. D., \& Garcia, V. X. P. (2006). Comportamento de 16 porta-enxertos para o tangor Murcott na região de Itirapina-SP. Revista Brasileira de Fruticultura, 28(1), 76-78.

Grizotto RK, Silva JA, Miguel FB, Modesto RT \& Vieira JB (2012). Qualidade de frutos de laranjeira Valência cultivada sob sistema tecnificado. Revista Brasileira de Engenharia Agricola e Ambiental - Agriambi. 16(7), 784-789.

Guimaraes, P. P., Botrel, R. T., Nogueira, N. W., Castro, V. G., Aguiar, G. P., \& Assis do Carmo, F. C. (2018). Forest products not timber the Brazilian Northeast-carnauba. Nativa 6(2) 213-218.

Instituto Adolfo Lutz - IAL. (2008). Métodos físicoquímicos para análise de alimentos (2010 pp.). São Paulo: Instituto Adolfo Lutz.

Instituto Nacional de Meteorologia - INMET. (2018). Banco de Dados Meteorológicos para Ensino e Pesquisa
- BDMEP. Recuperado em 27 de junho de 2018, de http:// www.inmet.gov.br/portal/index.php?r=bdmep/bdmep/

Kimball, D. A. (1991). Citrus processing: quality control and technology (453 pp.). New York: Van Nostrand Reinhold.

Koller, O. C. (1994). Citricultura: laranja, limão e tangerina (446 pp.). Porto Alegre: Rigel.

Lakatos, E. M., \& Marconi, M.A. (2017). Fundamentos de metodologia científica (8. ed., 368 pp.). São Paulo: Atlas.

Lemos, L. M. C., Siqueira, D. L., Salomão, L. C. C., Cecon, P. R., \& Lemos, J. P. (2012). Características físicoquímicas da laranja-pera em função da posição na copa. Revista Brasileira de Fruticultura, 34(4), 1091-1097.

Marconi, M. A., \& Lakatos, E. M. (2017). Técnicas de pesquisa: planejamento e execução de pesquisas, amostragens e técnicas de pesquisa, elaboração, análise e interpretação de dados (7. ed., 328 pp.). São Paulo: Atlas.

Passos, O. S., Soares Filho, W. S., Barbosa, C. J., \& Cunha Sobrinho, A. P. (2013). Clones da laranjeira 'DE RUSSAS' (4 pp.). Cruz das Almas: Embrapa Mandioca e Fruticultura.

Portella, C. R. (2015). Crescimento e produção inicial de cultivares de citros de mesa enxertadas sobre os portaenxertos 'Flying dragon'e limoeiro 'Cravo' (Dissertação de mestrado). Universidade Estadual do Norte Fluminense Darcy Ribeiro, Campos dos Goytacazes.

Rocha, R. H. C., Nascimento, S. R. C., Menezes, J. B., Nunes, G. H. S., \& Silva, E. O. (2005). Qualidade póscolheita do mamão formosa armazenado sob refrigeração. Revista Brasileira de Fruticultura, 27(3), 386-389.

Rodrigues, M. J. D. S., Ledo, C. A. S., Girardi, E. A., Almeida, L. A. H., \& Soares Filho, W. S. (2015). Fruit characterization and propagation of hybrid citrus rootstocks in protected environment. Revista Brasileira de Fruticultura, 37(2), 457-470.

Salibe, A. A. (1977). Curso de especialização em fruticultura: cultura de citros (3. ed., 188 pp.). Recife, SUDENE/UFRPe.

SAS Institute. (2017). SAS ${ }^{\circledR} 9.4$ Output Delivery System: User's Guide (1240 pp.). Cary: SAS institute.

Silva Júnior, B. G., Rocha, L. F., Amaral, F. H. C., Andrade, M. L., Falcão Neto, R., \& Cavalcante, Í. H. L. (2010). Laranja-da-terra: fruta cítrica potencial para o Piauí. Semina: Ciências Agrárias, 31(3), 557-562. 
Soares Filho, W. S., Medrado, A. M., Cunha, M. A. Sombra, K. E. S., Silva, A. C. C., Loureiro, F. L. C., \& P., Cunha Sobrinho, A. P., \& Passos, O. S. (2002). Uchôa, C. N. (2018). A citricultura como instrumento de Frequência de híbridos em cruzamentos controlados de citros: cultivo de sementes versus cultivo in vitro de embriões. Pesquisa Agropecuária Brasileira, 37(7), 981-988.

Sombra, K. E. S., Silva, A. C. C., Loureiro, F. L. C., \& Bastos, D. C. (2016). Citricultura desenvolvida na agricultura de base familiar do município de Russas, Ceará. Cultura Agronômica, 25(3), 303-316. preservação da agricultura familiar no semiárido cearense, Brasil. Revista de Extensão e Estudos Rurais, 7(1), 21-40.

Recebido: Junho 29, 2018

Aceito: Setembro 06, 2018

Como citar: Sombra, K. E. S., Silva, A. C. C., Rodrigues, A. J. O., Loureiro, F. L. C., Uchôa, C. N. \& Souza, P. A. (2018). Identificação e caracterização físico-química de frutos de laranja de Russas no semiárido cearense, Brasil. Citrus Research \& Technology, 39, e1035. https://doi.org/10.4322/crt.39161 